
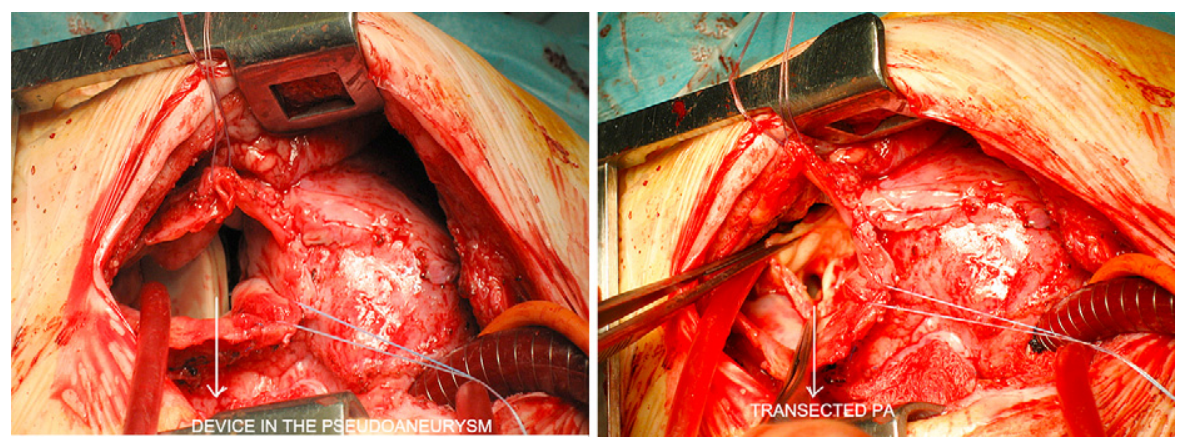

FIGURE 2. Intraoperative picture showing transection of the pulmonary artery and the device in the pulmonary artery lumen.

\section{DISCUSSION}

This case is reported to highlight the possibility of erosion and pseudoaneurysm formation after implantation of the FloWatch-PAB adjustable PA banding system. As far as we are aware, this is the second such complication with this device. ${ }^{2}$ The previous reported case had a similar complication, with pseudoaneurysm formation 7 weeks after the insertion. Doubts have been raised about the technical aspect of insertion being the cause for the pseudoaneurysm formation. ${ }^{1}$ In our case no intraoperative difficulty was reported. We therefore would like to highlight this specific occurrence of a pseudoaneurysm of the PA as a possible complication of the device rather than a problem with the technique of insertion. We would recommend anyone having this device inserted to have regular and frequent follow-up until the device is removed.

\section{References}

1. Corno AF, Pozzi M, Von Segesser LK. Flowatch and pseudoaneurysm: complication versus coincidence. J Thorac Cardiovasc Surg. 2006;131:928-9.

2. Michel-Behnke I, Akintuerk H, Valeske K, Thul J, Mueller M, Schranz D. Pseudoaneurysm of the pulmonary trunk after placement of an adjustable pulmonary artery banding device (FloWatch-PAB) in a patient with muscular ventricular septal defect. J Thorac Cardiovasc Surg. 2005;130:894-5.

\title{
An adult man presenting with hemoptysis caused by mature teratoma with rupture into the bronchus and pericardium and complicated by Haemophilus influenzae infection
}

\author{
Karthik Jothianandan, MD, ${ }^{\mathrm{a}}$ Amit S. Tibb, MD, ${ }^{\mathrm{b}}$ Michael McLemore, MD, ${ }^{\mathrm{c}}$ Steven Keller, $\mathrm{MD},{ }^{\mathrm{d}}$ and \\ David W. Appel, MD, ${ }^{\mathrm{b}}$ Bronx, NY
}

Mediastinal mature teratomas in adults are uncommon, typically asymptomatic tumors that arise in the anterior mediastinum. ${ }^{1}$ Usually, they are benign and resectable. ${ }^{1,2}$ Rarely, they rupture into adjacent cardiothoracic struc-

\footnotetext{
From the Departments of Internal Medicine, ${ }^{\mathrm{a}}$ Pulmonary Medicine, ${ }^{\mathrm{b}}$ Pathology, ${ }^{\mathrm{c}}$ and Cardiothoracic Surgery, ${ }^{\mathrm{d}}$ Montefiore Medical Center, Bronx, NY. Disclosures: None.

Received for publication Feb 20, 2009; accepted for publication March 7, 2009; available ahead of print May 11, 2009.

Address for reprints: Karthik Jothianandan, MD, Internal Medicine, Montefiore Medical Center, 111 E 210 St, Bronx, NY 10467 (E-mail: karthik.jothi@gmail.com).

J Thorac Cardiovasc Surg 2010;139:e104-7 $0022-5223 / \$ 0.00$

Published by Elsevier Inc. on behalf of The American Association for Thoracic Surgery

doi:10.1016/j.jtcvs.2009.03.003
}

tures. ${ }^{1,2}$ Hemoptysis as a presenting symptom is rare. Among adults, bacterial infection complicating mediastinal mature teratoma rupture has not been described.

Herein we report the case of a 58-year-old man who presented with hemoptysis and was found to have an unresectable mediastinal mature teratoma with rupture into a bronchus, intrapericardial tumor extension surrounding the left and right ventricles, and simultaneous Haemophilus influenzae infection. A mediastinal mature teratoma in an adult with this complex presentation has not been described previously to our knowledge.

\section{CLINICAL SUMMARY}

A 58-year-old man from Ghana was hospitalized after 5 days of hemoptysis, cough, dyspnea, and pleuritic left chest 


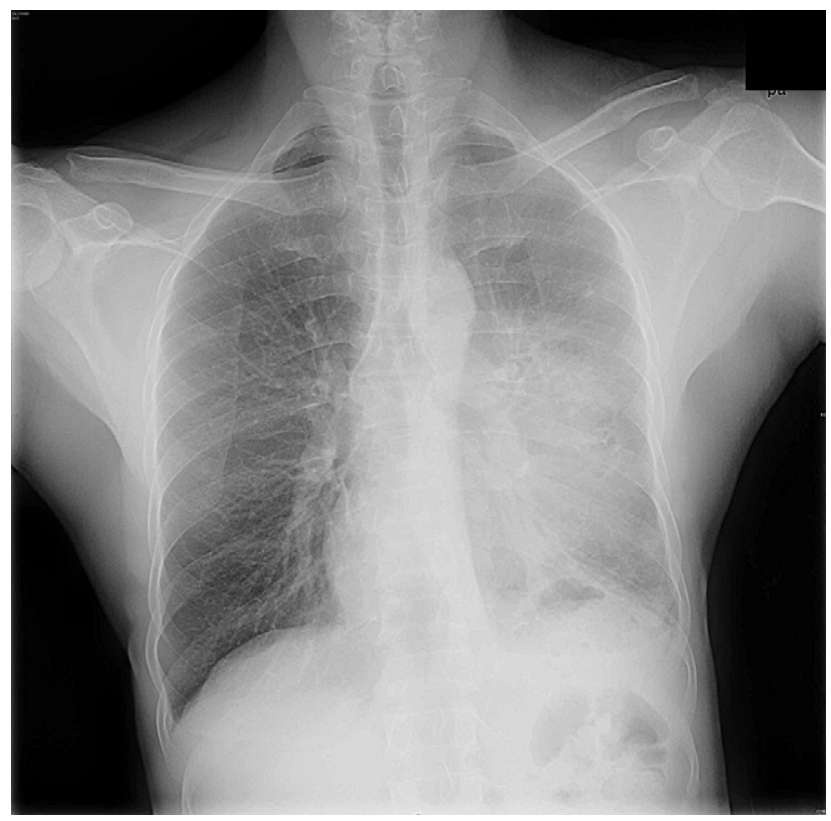

FIGURE 1. Large opacity with multiloculated lucencies superior and adjacent to the left heart border.

pain. Twelve years earlier, he was treated for pneumonia. The left upper hemithorax revealed reduced tactile fremitus, dullness, and monophonic rhonchi. Routine laboratory test results were normal. A tuberculin skin test showed a nonreactive result. Sputum smears and cultures revealed no tuberculosis. Blood cultures were without growth. Chest radiographic analysis revealed a large opacity with multiloculated lucencies superior and adjacent to the left heart border (Figure 1). Noncontrast computed tomographic analysis of the chest showed a $6.2 \mathrm{~cm} \times 7.0 \mathrm{~cm} \times 4.0 \mathrm{~cm}$ necrotic, cavitating, heterogeneous anterior mediastinal mass containing fat, soft tissue, and gas that suggested bronchial rupture. The mass appeared to occlude the anterior segmental bronchus of the left upper lobe, invade the left hilum posteriorly, and extend directly into the pericardium (Figure 2).

Fiberoptic bronchoscopy revealed left upper lobe bronchial edema and, at its origin, left lower lobe narrowing caused by external compression. All segmental and subsegmental bronchi were patent and without lesions. Transthoracic needle biopsy specimens of the mass demonstrated thymic tissue, respiratory epithelium, and both apocrine and eccrine sweat glands, which are consistent with a mature cystic teratoma (Figure 3). No malignant cells were identified. The mediastinal mature teratoma tissue grew $\mathrm{H}$ influen$z a e$. During median sternotomy, a tumor was seen infiltrating through the ruptured pericardium and around both ventricles. The left anterior descending coronary artery passed through the mass. Complete dissection of the tumor from the heart was impossible. Hemoptysis, chest pain, and dyspnea subsided during treatment with piperacillin-tazobactam. Tumor resection during cardiopulmonary bypass, reconstruction of

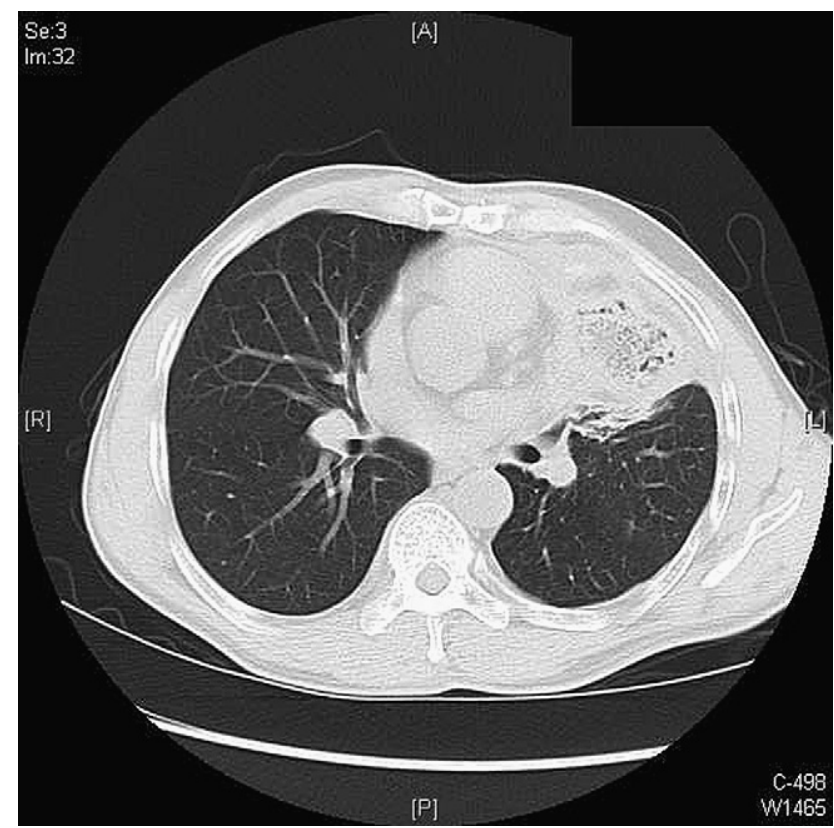

FIGURE 2. Necrotic cavitating anterior mediastinal mass containing fat, soft tissue, and gas, occluding a bronchus and invading into pericardium.

the loculated ventricles, and heart-lung transplantation were discussed with the patient. At the time, however, he felt well and declined further surgical intervention. Repeat chest computed tomograms taken 6 months later showed no significant interval change. Currently, 12 months after sternotomy, the patient feels well and is without symptoms.

\section{DISCUSSION}

Common causes of anterior mediastinal masses include thymoma, thymic carcinoma, thymic carcinoid tumor, thymolipoma, germ cell tumors, parathyroid adenoma, and lymphoma. ${ }^{1}$ Nonneoplastic conditions include thymic cyst, lymphangioma, and intrathoracic thyroid tissue. ${ }^{1}$

Mediastinal germ cell tumors represent approximately $10 \%$ to $15 \%$ of adult anterior mediastinal tumors, and of these, mature teratomas are the most common. ${ }^{1,3}$ Mediastinal mature teratomas are histologically well differentiated and benign, containing varying amounts of tissues derived from at least 2 of 3 primitive germ layers: ectoderm, mesoderm, and endoderm. ${ }^{1-3}$ Mediastinal mature teratomas are seen in adults age 20 to 40 years and have equal sex distribution. ${ }^{1,3}$ Typically, these tumors are cystic when in the mediastinum and solid when elsewhere. ${ }^{4}$ Twenty-five percent of mediastinal mature teratomas undergo malignant degeneration, regardless of the presence or absence of immature elements. ${ }^{4}$

In $60 \%$ of cases of mediastinal mature teratomas, patients presented asymptomatically, and tumors were discovered incidentally. ${ }^{2}$ When symptomatic, patients presented with cough $(60 \%)$, chest pain $(30 \%)$, fevers/chills $(20 \%)$, and dyspnea $(16 \%){ }^{2}$ Trichoptysis (expectoration of hair or sebaceous debris) was very rare but pathognomonic for 


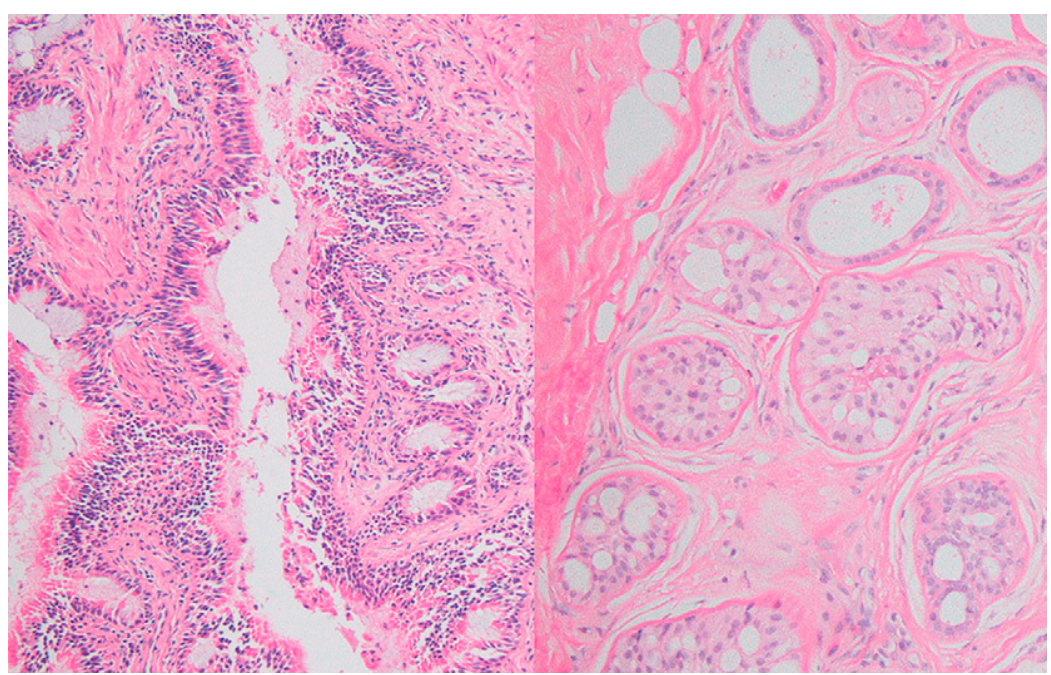

FIGURE 3. Biopsy showing respiratory epithelium (left) and apocrine and eccrine sweat glands (right).

a teratoma. ${ }^{1,2}$ Direct tumor compression on adjacent structures might cause respiratory failure from airways obstruction, dysphagia, diaphragmatic paralysis, Horner syndrome, and superior vena cava syndrome. ${ }^{2}$ Choi and colleagues ${ }^{5}$ demonstrated that severe symptoms (chest pain or hemoptysis) were more commonly found in tumors that ruptured into adjacent structures $(71 \%)$ than in unruptured tumors. Functionally active tumors have caused sebaceous secretion, insulin production, chorionic gonadotropin secretion, or exocrine secretion by pancreatic, salivary, or intestinal tissue. ${ }^{5}$

The mechanism of rupture is thought to be secondary to proteolytic or digestive enzymes secreted by intestinal mucosa or pancreatic tissue found in the teratoma that leads to inflammation and necrosis of adjacent organs. ${ }^{1,2,6} \mathrm{En}$ zyme-induced erosions have caused tumor rupture into the tracheobronchial tree, pleural cavity, vascular structures, pericardium, and lung parenchyma and even through the skin to form a draining fistula. ${ }^{1,2,4,7}$

Hemoptysis is a rare presentation of mediastinal mature teratoma and usually connotes rupture. Choi and colleagues ${ }^{5}$ found that 1 of their 7 patients with ruptured mediastinal teratomas presented with hemoptysis. Gunes and associates ${ }^{8}$ reported the case of a 33-year-old-man presenting with massive hemoptysis caused by a mediastinal mature teratoma rupturing into the bronchus. Robertson and coworkers ${ }^{9}$ described an 8-month-old infant with an anterior mediastinal teratoma presenting with life-threatening hemoptysis as a result of gastric mucosa of the tumor causing ulceration into the lung parenchyma. Both Steier ${ }^{10}$ and Bandyopadhyay and colleagues ${ }^{11}$ described patients with mediastinal mature teratomas and recurrent hemoptysis. Hayden and associates ${ }^{12}$ reported a patient with a benign intrathoracic teratoma who presented with hemoptysis and who was found to have extensive pleural adhesions and systemic-pulmonary artery shunting. Chen and coworkers ${ }^{13}$ described 2 patients presenting with recurrent hemoptysis and trichoptysis with mediastinal mature teratomas.

Rupture of the mediastinal mature teratoma through the pericardium is an even rarer complication. Presentation with life-threatening cardiac tamponade ${ }^{14-17}$ and pericarditis ${ }^{18}$ has been described.

A few cases of anterior mediastinal masses with simultaneous bacterial infection have been reported in children but not in adults. Yu and colleagues ${ }^{19}$ described a 13-year-old boy with fever and cough but no hemoptysis who had a mediastinal mature teratoma with rupture into the pleura, lung, and bronchus complicated by Mycoplasma pneumoniae infection. Youngson and associates ${ }^{20}$ described a 15-monthold boy presenting with respiratory distress who was found to have a thymic cyst with $H$ influenzae abscess formation.

Radiologically, mediastinal mature teratomas are round to lobulated, well-defined anterior mediastinal masses that typically protrude to one side of the midline and can reach large sizes. ${ }^{1,7}$ Up to $26 \%$ show calcification and rarely show teeth or bone on chest radiographic analysis. ${ }^{1,7}$ On chest computed tomographic analysis, mediastinal mature teratomas are often mulitlocular cystic tumors with variably thick walls. The combination of fluid, soft tissue, calcium, and/ or fat attenuation is highly specific for a mediastinal mature teratoma. ${ }^{1,2}$ Chest computed tomography, magnetic resonance imaging, or both are often useful to help further localize these lesions and to confirm rupture into adjacent structures. ${ }^{1,2,4,5}$ Choi and colleagues ${ }^{5}$ found that all ruptured mediastinal teratomas showed inhomogeniety, whereas $90 \%$ of unruptured masses showed homogeneous densities.

Because $25 \%$ of mediastinal mature teratomas undergo malignant degeneration, complete surgical resection is the treatment of choice and is curative. ${ }^{2,4}$ Surgical management of ruptured tumors, however, is often more complicated than that of unruptured tumors because the internal 
components of the teratoma leak into the thoracic cavity, causing inflammation and adhesions. ${ }^{5}$ When benign teratomas cannot be excised completely without endangering vital surrounding structures, subtotal resection can relieve compressive symptoms. ${ }^{2}$

Our patient with a mediastinal mature teratoma is unique because (1) he presented with hemoptysis (rare) and (2) he had bacterial infection of the mediastinal mature teratoma (not described before in adults). Hemoptysis and chest computed tomographic evidence of gas in the mediastinal mature teratoma reflected tumor and tracheobronchial tree rupture.

Rupture could have resulted from infection of the mediastinal mature teratoma with $H$ influenzae, release of proteolytic enzymes from pancreatic/intestinal mucosal tissues in the tumor, or both. ${ }^{1,2,6} \mathrm{We}$ found no evidence of infection beyond the mediastinal mature teratoma itself to explain its rupture, such as pneumonia, and therefore we speculate that rupture resulted from mediastinal mature teratoma proteolytic enzyme release and led to hemoptysis and secondary mediastinal mature teratoma infection. Pancreatic tissue, the most common kind found in mediastinal mature teratomas, ${ }^{6}$ was not found in our needle biopsy specimen, but our specimen was small, and it is likely that the unsampled tumor contained pancreatic/intestinal mucosal tissue.

Even though the mediastinal mature teratoma in our patient was benign and histologically mature, complete resection, our recommended treatment of choice, ${ }^{1}$ was impossible because the mediastinal mature teratoma had infiltrated the pericardium and encased both ventricles and the coronary arteries. At the time, we discussed with our patient tumor resection during cardiopulmonary bypass, reconstruction of the loculated ventricles, and heart-lung transplantation. He felt well and was wary of the attendant risks of these procedures and declined surgical intervention. Although such intervention remains a future consideration, 12 months after his presentation, he feels well and is fully active.

\section{References}

1. Strollo DC, Rosado-de-Christenson ML, Jett JR, et al. Primary mediastinal tumors: part 1. Tumors of the anterior mediastinum. Chest. 1997;112:511-22.

2. Duwe BV, Sterman DH, Musani AI. Tumors of the mediastinum. Chest. 2005; 128:2893-909.

3. Nichols CR. Mediastinal germ cell tumors: clinical features and biologic correlates. Chest. 1991;99:472-9.

4. Cohn WE. Anterior mediastinal masses. UpToDate 2008. Available at: http://www utdol.com/online/content/topic.do?topicKey=plum_dxs/6444\&selectedTitle= $1 \sim 15 \&$ source $=$ searchresult. Accessed 29 March 2009.

5. Choi SJ, Lee JS, Song KS, et al. Mediastinal teratoma: CT differentiation of ruptured and unruptured tumors. Am J Roentgenol. 1998;171:591-4.

6. Southgate J, Slade PR. Teratodermoid cyst of the mediastinum with pancreatic enzyme secretion. Thorax. 1982;37:476-7.

7. Lewis BD, Hurt RD, Payne WS, et al. Benign teratoma of the mediastinum. J Thorac Cardiovasc Surg. 1983;86:727-31.

8. Gunes S, Varon J, Walsh G. Mediastinal teratoma presenting as massive hemoptysis in an adult. J Emerg Med. 1997;15:313-6.

9. Robertson JM, Fee HJ, Mulder DG. Mediastinal teratoma causing life-threatening hemoptysis. Its occurrence in an infant. Am J Dis Child. 1981;135:148-50.

10. Steier KJ. Benign cystic teratoma of the lung. Postgrad Med. 1988;83:85-6.

11. Bandyopadhyay SK, Bandyopadhyay R, Moulick A, et al. Benign mediastinal teratoma producing recurrent hemoptysis. J Assoc Physicians India. 2005;53: 698.

12. Hayden CK Jr, Swischuk LE, Schwartz MZ, et al. Systemic-pulmonary shunting and hemoptysis in a benign intrathoracic teratoma. Pediatr Radiol. 1984; $14: 52-4$.

13. Chen RF, Chang TH, Chang CC, et al. Mediastinal teratoma with pulmonary involvement presenting as hemoptysis: two cases [abstract]. Chest. 2007;132: 683a.

14. Prabhakar G, Nigam BK, Williams WB. Benign mediastinal teratoma causing pericardial tamponade and pleural effusion. Eur J Cardiothorac Surg. 1987;1: 53-4.

15. Oomman A, Santhosham R, Vijayakumar C, et al. Anterior mediastinal teratoma presenting as cardiac tamponade. Indian Heart J. 2004;56:64-6.

16. Sakamoto K, Kase M, Mo M, et al. Mediastinal mature teratoma perforated into the pericardial sac: a case report. Kyobu Geka. 2000;53:74-7.

17. Yoshida K, Yamanda T, Aoki T, et al. A case of mature teratoma perforated into the pericardial cavity. Nippon Kyobu Geka Gakkai Zasshi. 1997;45: 1107-10.

18. Massari FM, La Marchesina U, Buffa P, et al. Benign mediastinal teratoma with pericardial symptoms. The utility of echocardiography in diagnosis. G Ital Cardiol. 1997;27:476-9.

19. Yu CW, Hsieh MJ, Hwang KP, et al. Mediastinal mature teratoma with complex rupture into the pleura, lung, and bronchus complicated with mycoplasma pneumonia. J Thorac Cardiovasc Surg. 2007;133:1114-5.

20. Youngson GG, Ein SH, Geddie WR, et al. Infected thymic cyst: an unusual cause of respiratory distress in a child. Pediatr Pulmonol. 1987;3:276-9. 